\title{
Most mammalian mRNAs are conserved targets of microRNAs
}

\author{
Robin C. Friedman, ${ }^{1,2,3}$ Kyle Kai-How Farh, ${ }^{1,2,4}$ Christopher B. Burge, ${ }^{1,5}$ \\ and David P. Bartel ${ }^{1,2,5}$

\begin{abstract}
${ }^{1}$ Department of Biology, Massachusetts Institute of Technology, Cambridge, Massachusetts 02139, USA; ${ }^{2}$ Whitehead Institute for Biomedical Research and Howard Hughes Medical Institute, Cambridge, Massachusetts 02142, USA; ${ }^{3}$ Computational and Systems Biology Program, Massachusetts Institute of Technology, Cambridge, Massachusetts 02139, USA; ${ }^{4}$ Division of Health Sciences and Technology, Massachusetts Institute of Technology, Cambridge, Massachusetts 02139, USA
\end{abstract}

\begin{abstract}
MicroRNAs (miRNAs) are small endogenous RNAs that pair to sites in mRNAs to direct post-transcriptional repression. Many sites that match the miRNA seed (nucleotides 2-7), particularly those in 3' untranslated regions (3'UTRs), are preferentially conserved. Here, we overhauled our tool for finding preferential conservation of sequence motifs and applied it to the analysis of human 3'UTRs, increasing by nearly threefold the detected number of preferentially conserved miRNA target sites. The new tool more efficiently incorporates new genomes and more completely controls for background conservation by accounting for mutational biases, dinucleotide conservation rates, and the conservation rates of individual UTRs. The improved background model enabled preferential conservation of a new site type, the "offset 6mer," to be detected. In total, $>45,000$ miRNA target sites within human 3'UTRs are conserved above background levels, and $>60 \%$ of human protein-coding genes have been under selective pressure to maintain pairing to miRNAs. Mammalian-specific miRNAs have far fewer conserved targets than do the more broadly conserved miRNAs, even when considering only more recently emerged targets. Although pairing to the $3^{\prime}$ end of miRNAs can compensate for seed mismatches, this class of sites constitutes less than $2 \%$ of all preferentially conserved sites detected. The new tool enables statistically powerful analysis of individual miRNA target sites, with the probability of preferentially conserved targeting $\left(P_{C T}\right)$ correlating with experimental measurements of repression. Our expanded set of target predictions (including conserved 3 '-compensatory sites), are available at the TargetScan website, which displays the $P_{C T}$ for each site and each predicted target.
\end{abstract}

[Supplemental material is available online at www.genome.org.]

MicroRNAs are 22 -nucleotide (nt) endogenous RNAs that derive from distinctive hairpin precursors in plants and animals (Bartel 2004). After incorporation into a silencing complex, which contains at its core an Argonaute protein, an miRNA can pair to an mRNA and thereby specify the post-transcriptional repression of that protein-coding message, either by transcript destabilization, translational repression, or both. MicroRNAs constitute one of the more abundant classes of gene-regulatory molecules in animals, with hundreds of distinct miRNAs confidently identified in both human and mouse (Landgraf et al. 2007). A central goal for understanding the functions of all these small regulatory RNAs has been to determine which messages are targeted for repression.

The search for biological targets of metazoan miRNAs has benefited greatly from the comparative analysis of orthologous mRNAs. Targets of miRNAs can be predicted above the background of false-positive predictions by requiring conserved Watson-Crick pairing to the $5^{\prime}$ region of the miRNA, known as the miRNA seed (Lewis et al. 2003). Because so many messages have preferentially preserved their pairing to miRNA seeds, targets can be predicted simply by searching for conserved 6-8mer matches to miRNA seed region (Brennecke et al. 2005; Krek et al. 2005;

${ }^{5}$ Corresponding authors.

E-mail dbartel@wi.mit.edu; fax (617) 258-6768.

E-mail cburge@mit.edu; fax (617) 452-2936.

Article published online before print. Article and publication date are at http:// www.genome.org/cgi/doi/10.1101/gr.082701.108. Freely available online through the Genome Research Open Access option.
Lewis et al. 2005). Four types of seed-matched sites are known to be selectively conserved (Lewis et al. 2005): the 6mer site, which perfectly matches the 6-nt miRNA seed, the 7 mer-m8 site, which comprises the seed match supplemented by a Watson-Crick match to miRNA nucleotide 8 , the 7 mer-A1 site, which comprises the seed match supplemented by an A across from miRNA nucleotide 1 , and the 8 mer site, which comprises the seed match supplemented by both the m8 and the A1 (Fig. 1A). Supporting the validity of seed-matched target predictions, cellular messages that either decrease following miRNA addition or increase following miRNA disruption preferentially contain seed matches (Lim et al. 2005; Giraldez et al. 2006; Rodriguez et al. 2007), with the following hierarchy of site efficacy: $8 \mathrm{mer}>7 \mathrm{mer}-\mathrm{m} 8>7 \mathrm{mer}-$ A1 > 6mer (Grimson et al. 2007; Nielsen et al. 2007). The same is true when examining protein levels (Baek et al. 2008; Selbach et al. 2008).

In addition to its utility for predicting the identities of the regulatory targets, comparative sequence analysis has provided fundamental insights regarding features of mRNA sites required for effective miRNA recognition. For example, a systematic analysis of matches to 7-nt segments spanning the length of the miRNAs showed that only those matching the $5^{\prime}$ region of the miRNA are conserved more than expected by chance, thereby defining the seed region as the key determinant of miRNA specificity (Lewis et al. 2003). Additional analyses of preferential conservation uncovered the importance of non-Watson-Crick recognition of an $\mathrm{A}$ across from miRNA nucleotide 1 and of an A or $\mathrm{U}$ across from 
nucleotide 9 (Lewis et al. 2005; Nielsen et al. 2007). Comparative analyses revealed targeting in open reading frames (ORFs) (Lewis et al. 2005; Stark et al. 2007a) but also supported experimental findings that sites are more effective if they fall outside the path of the ribosome (Grimson et al. 2007). Comparative analyses supported the importance of other features of site context, including

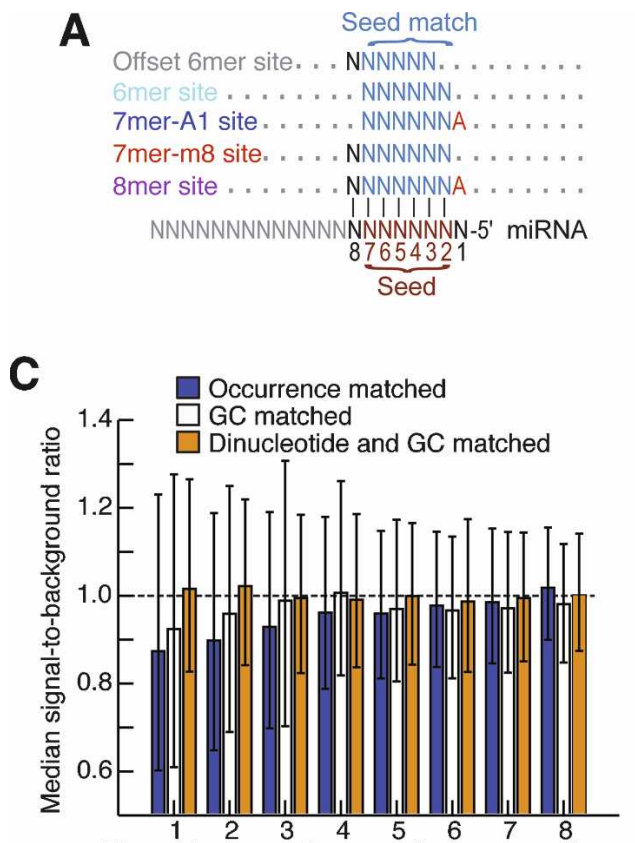

Binned by increasing expected conservation

D

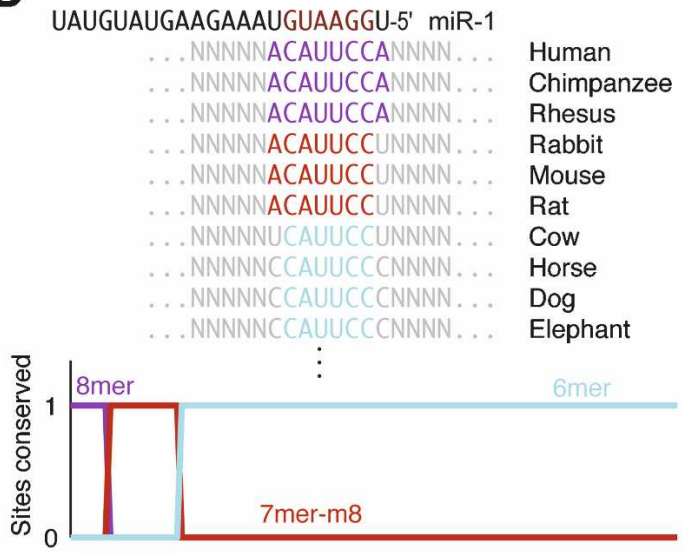

Branch-length cutoff
B
miR-1 8mer site in SLC35B4

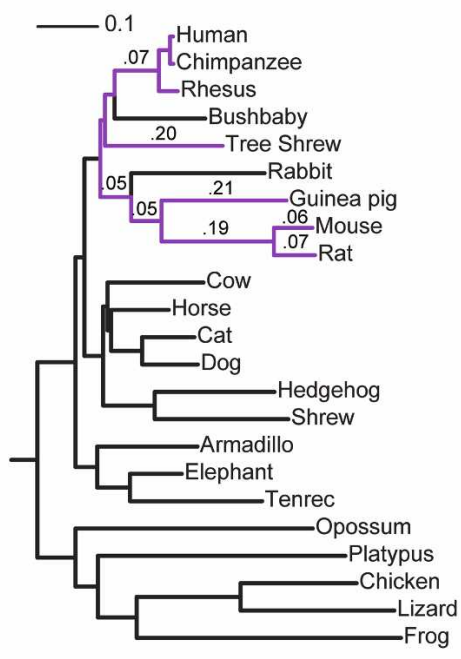

Branch length $=0.07+0.20+0.05+0.05$ + six smaller branch lengths $=1.0$

\section{E}

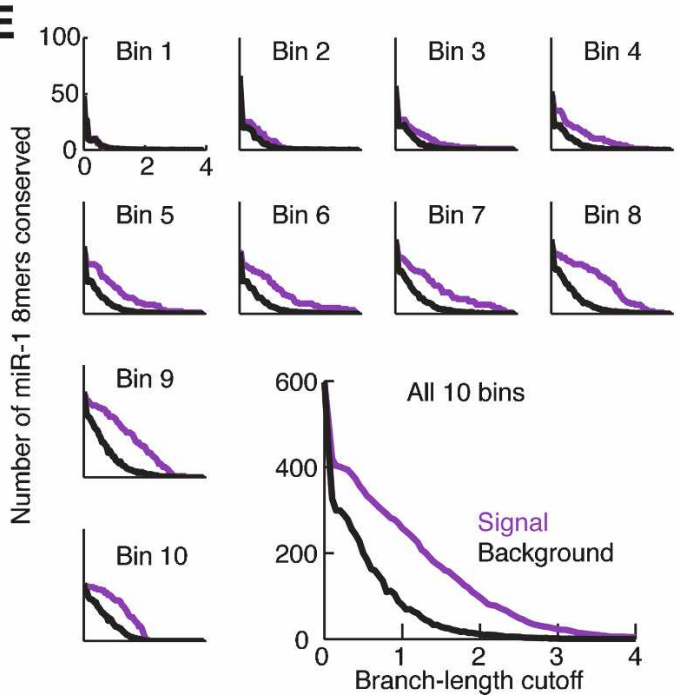

Figure 1. Method for detecting preferential conservation of miRNA sites. ( $A$ ) Sites matching the miRNA seed region. All four canonical sites (colored) share six contiguous Watson-Crick matches to the miRNA seed (nucleotides 2-7); the offset 6mer contains six contiguous matches to nucleotides 3-8. (B) Phylogenetic conservation of individual sites. Each panel represents a miR-1 8mer site conserved to a branch length of 1.0. The 3'UTR of SLC35B4 falls into the fourth-most poorly conserved bin, which has a phylogeny with relatively long branch lengths (left), whereas the 3'UTR of SPRED1 falls into the most well-conserved bin, which has a phylogeny with shorter branch lengths (right). Branch segments connecting the species with sites are colored purple, with numbers indicating the lengths of the longer segments. The lengths of the segments connecting the species are summed to yield the branch-length score (equation). (C) Performance of controls matched for number of occurrences in human 3'UTRs, GC content, or expected conservation as calculated by a first-order Markov model. For each possible RNA 7 mer, conservation rates (signal) and average conservation rates for 50 control 7mers (background) were calculated for all 3'UTRs at a branch-length cutoff of 1.0. Error bars indicate 25th and 75th percentiles. Because most 7mer motifs are not selectively maintained, well-performing controls should yield median signal-to-background ratios near 1.0 , with low variability for every bin. $(D)$ Nested site conservation. Because the seed-matched sites are interrelated, we subtract conserved instances of extended sites from those of shorter sites. This hypothetical site is an 8mer match to miR-1 in a human 3'UTR that is more broadly conserved as a $7 \mathrm{mer}-\mathrm{m} 8$ than as an $8 \mathrm{mer}$ site, and as a 6 mer than as a 7 mer-m8 site. As a result of nested subtraction, our method considers this site an 8 mer at low branch-length cutoffs but not a $6 \mathrm{mer}$ or $7 \mathrm{mer}$. At moderate branch-length cutoffs, it switches to a conserved $7 \mathrm{mer}-\mathrm{m} 8$ site, and at high branch-length cutoffs it switches to a conserved $6 \mathrm{mer}$ site but not a $7 \mathrm{mer}$ or $8 \mathrm{mer}$. (E) Signal and background for the miR-1 8mer site. For each UTR bin 1 through 10, with bin 1 having the least conserved UTRs and bin 10 the most conserved, the number of miR-1 sites conserved at the indicated branch-length cutoff is plotted with estimated background (small plots). Results for all 10 bins were then combined to represent the aggregate signal and background for this site (large plot). 
positioning within high local AU composition (Grimson et al 2007; Nielsen et al. 2007), away from the centers of long UTRs (Gaidatzis et al. 2007; Grimson et al. 2007; Majoros and Ohler 2007), and near to nucleotides that can pair to miRNA nucleotides 13-16 (Grimson et al. 2007).

Comparative analysis has also revealed the wide scope of metazoan miRNA targeting, indicating that many genes of mammals, flies, and worms are miRNA targets (Brennecke et al. 2005; Krek et al. 2005; Lewis et al. 2005; Xie et al. 2005; Lall et al. 2006). For example, combining the 3 'UTR conservation attributed to miRNA seed matching with that from ORFs indicates that over one third of human protein-coding genes have been under selective pressure to maintain pairing to miRNAs (Lewis et al. 2005). Moreover, the selective depletion of seed-matching sites in messages highly expressed in the same tissues as the miRNAs implies frequent nonconserved targeting (Farh et al. 2005; Stark et al. 2005).

Ever since the availability of whole-genome multiple alignments (Blanchette et al. 2004), sites have been considered conserved if they are retained at orthologous locations in every genome under consideration and considered "nonconserved" or poorly conserved if they are missing or have changed in one of the genomes. This binary approach has been very productive but becomes less suitable now that the alignments include more than a few genomes. Requiring conservation in every species of a 28genome alignment would exclude sites that are under strong selective pressure to be conserved in many genomes yet are missing at the orthologous position in some genomes either because of lineage-specific loss, gain, or substitution, or because of imperfections in sequencing, assembly, or alignment. To capture more of the conserved sites, a quantitative approach has been developed that makes the reasonable assumption that aligned sites within orthologous genes have a single origin and measures the portion of the phylogenetic tree that retains each site by summing the branch length over which each site has been preserved (Kheradpour et al. 2007). Because it represents an estimate of the amount of evolutionary time over which a site has been conserved, this branch-length score yields a multivalued metric that accounts for phylogenetic relationships between the species studied (Kheradpour et al. 2007). The score is interpreted by selecting a branch-length cutoff that separates more conserved and less conserved sites. Sliding the branch-length cutoff from zero to the total length of all branches enables tuning of sensitivity and specificity. This method has been applied to the 12-genome alignments of flies to predict conserved miRNA sites with sensitivity substantially improved over the previous binary approach (Kheradpour et al. 2007; Ruby et al. 2007) but has yet to be applied to mammalian site conservation.

While whole-genome alignments have made it simple to detect the conservation of sites in orthologous locations of genes, it is much more difficult to distinguish those sites or motifs under selective pressure to be maintained from those conserved by chance. A general attempt to detect preferential conservation of any motif used a simple $Z$-score test but did not control for genomic location or sequence characteristics (Xie et al. 2005). Another approach, developed for detecting maintenance of miRNA sites, has been to generate cohort sets of miRNA-like sequences, then determine the number of conserved sites that match these control sequences and use this as the estimate of chance conservation (Lewis et al. 2003). When choosing these controls carefully so as to avoid sites underrepresented in mRNA sequences, this approach has been effective for evaluating sets of miRNA sites in aggregate (Lewis et al. 2003, 2005; Brennecke et al. 2005; Krek et al. 2005; Stark et al. 2005; Lall et al. 2006; Kheradpour et al. 2007; Ruby et al. 2007). As previously implemented, however, this approach breaks down when examining individual miRNA-site interactions because of a failure to account adequately for differing mutational biases, dinucleotide conservation rates, and local conservation rates.

Here we develop an improved method for quantitatively evaluating site conservation and apply it to the study of vertebrate miRNA targeting. The improved sensitivity uncovered classes of sites, including offset seed matches and 3 '-compensatory sites, whose conservation previously had not been detected with confidence. Overall, we find three times as many preferentially conserved sites as detected previously, thereby increasing the known scope and density of conserved miRNA regulatory interactions.

\section{Results and Discussion}

\section{Detection of seed match conservation with increased sensitivity and statistical power}

When using a branch-length metric to evaluate motif conservation, the first step is to build a phylogenetic tree based on the genomic regions under investigation (Kheradpour et al. 2007), which in our case was 3'UTRs. One major innovation of our method was to build the phylogeny in a way that controlled for the conservation of individual UTRs. Because mutation, gene conversion, and crossover rates vary throughout the genome (Wolfe et al. 1989; Hwang and Green 2004; Kauppi et al. 2004), different UTRs have substantially different background conservation levels. Moreover, some vertebrate genomes have low coverage and are missing a substantial fraction of genes, also affecting the apparent background conservation. Most methods for detecting positive selection take into account local conservation rates (Yang and Bielawski 2000) (for example, $K_{\mathrm{s}}$ in $K_{\mathrm{a}} / K_{\mathrm{s}}$ ), but genome-scale methods for detecting purifying selection have thus far not accounted for this factor. In addition to differences in basal conservation rates, UTRs have sequence-dependent functions apart from miRNAs, which can influence conservation levels. A site falling within a UTR with high overall conservation is far less likely to be conserved due to miRNA targeting than is one falling within a rapidly evolving UTR (Lewis et al. 2005). Any method that treats all the UTRs the same greatly overestimates purifying selection of sites in well-conserved UTRs and underestimates purifying selection of sites in poorly conserved UTRs.

Starting with a 28-way vertebrate whole-genome alignment that included 18 placental mammals and 10 other vertebrates (Miller et al. 2007), we extracted the human 3'UTRs and homologous regions from the 22 non-fish genomes. The five fish genomes were excluded because they lacked a sufficient amount of aligned 3'UTR sequence. To help control for individual UTR conservation, 3'UTRs were separated by conservation rate into 10 equally sized bins, and a unique set of branch lengths based on 3'UTR sequence alignments was constructed for each bin (Fig. 1B; Supplemental Fig. 1). The conservation of a given sequence (e.g., an 8mer miR-1 site in a particular 3'UTR) was then assessed by summing the total branch length in the phylogenetic tree connecting the subset of species having the sequence perfectly aligned, using the tree representing the bin of the 3'UTR under investigation. This branch-length value had no units, with a value of 1.0 corresponding to the average conservation of a single nucleotide in similar UTRs, and thus resembled a non-

\section{Genome Research}

www.genome.org 
normalized version of the branch-length score described by Kheradpour et al. (2007). In our analyses, however, a site in a more divergent UTR needed to be conserved in fewer orthologs to achieve the same branch-length value because the branch lengths in a phylogeny representing the more divergent UTRs were longer than those of one representing more conserved UTRs. For example, the 8mer miR-1 site found within in the human SLC35B4 UTR received the same value as the site within the SPRED1 UTR, even though it was present in fewer aligned genomes (Fig. 1B).

Because sequences can be conserved by chance or for many reasons other than functional miRNA targeting, the branchlength values were only interpretable when considered within the context of the estimated background conservation. Our method attempted to control for many factors that can affect the conservation level of a short sequence of length $k$ (a $k$-mer), including GC content, dinucleotide content, the interrelation of miRNA seed-match types, genome alignment quality, and the local conservation rate. The combined effects of all of these factors on background conservation were estimated based on empirically observed conservation of $k$-mers as opposed to theoretical calculations. As done previously (Lewis et al. 2003, 2005; Brennecke et al. 2005; Krek et al. 2005; Stark et al. 2005; Lall et al. 2006; Kheradpour et al. 2007; Ruby et al. 2007), the expected fraction of sites conserved due to miRNA recognition was estimated using a cohort of $k$-mers with similar properties, which were presumed to be subject to the same evolutionary pressures except for the possible miRNA regulatory relationship. Because we did not allow conserved $k$-mers that were seed matches for miRNAs with any known conservation, and because the discovery rate of highly conserved vertebrate miRNAs has dropped dramatically in recent years, the control $k$-mers can be assumed devoid of conservation due to miRNA targeting. Three substantial improvements to the estimation of background conservation were introduced. First, we matched control $k$-mers using an expected conservation based on both the $k$-mer's GC content and the expected conservation of its constituent dinucleotides, which enabled a more rigorous and accurate estimate of background conservation levels for individual miRNAs (Fig. 1C). Previous methods matched $k$-mers based on their abundance in human 3'UTRs, which is adequate when analyzing large groups of miRNAs, but this variable is poorly correlated to conservation for individual miRNAs (Fig. 1C) and can be affected by evolutionary avoidance of $k$-mers, a known property of miRNA seed matches (Farh et al. 2005; Stark et al. 2005). Second, we created mutually exclusive seed-match classes by subtracting the signal and the background of larger seed matches (e.g., 8mers) from the smaller seed matches that could be contained within them (e.g., 7mers, Fig. 1D). This protected against double-counting conservation while increasing sensitivity by more closely matching control $k$-mer sizes to the observed conservation (see Supplemental material for discussion). Third, the estimate of background conservation controlled for the conservation of individual UTRs, in that control cohorts were analyzed using the same 10 phylogenetic trees and the same 10 UTR data sets were employed for analysis of authentic sites. Without this improvement, different members of the control cohort had widely varying conservation. By reducing this variability, more precise background estimates were achieved, which enabled more sensitive detection of site conservation. Thus, we calculated 10 distributions of branch-length values for both signal and background using both the $k$-mer and its set of controls and then summed these distributions to compile the overall signal and background distributions for each $k$-mer (Fig. 1E; Supplemental Discussion). These three innovations all helped to control for the background conservation specific to individual seed-match sites, enabling statistically sound comparisons between the conservation of seed-match types, between seed matches to different miRNAs, and even between individual sites.

\section{At least 44,000 sites are selectively maintained because of miRNA targeting}

We first looked for excess conservation of seed matches for a set of highly conserved miRNAs that appear to have been present since the last common ancestor of all 23 vertebrate species under consideration, defined as those mammalian miRNAs also found in chicken, lizard, or fish, which fell into 87 families based on the identity of nucleotides 2-8 (Supplemental Table 1). For each $k$ mer, representing a single seed-match type for a particular miRNA, the distribution of branch-length values was compiled for sites present in human 3'UTRs. As the branch-length-value cutoff was increased from zero, the number of sites that matched control sequences decreased faster than did the number matching authentic miRNA seed matches (Fig. 2A). At any particular branch-length cutoff, if the number of conserved sites of a $k$-mer (the signal) was higher than that of control sequences (the background), the excess conservation was attributed to purifying selection. We use the term "background" instead of "noise" because the latter term may connote variance in the background estimate as opposed to the estimate itself. The number of sites conserved above background reflects the sensitivity of the analysis, whereas the ratio of signal to background reflects its specificity.

We first considered the three 7-8mer seed-match types (8mer, 7mer-m8, 7mer-A1), which correlate most strongly with targeting efficacy (Grimson et al. 2007; Nielsen et al. 2007) and are among the miRNA matches currently used to predict conserved targets of metazoan miRNAs (Fig. 2B; Brennecke et al. 2005; Grun et al. 2005; Krek et al. 2005; Lewis et al. 2005; Lall et al. 2006; Ruby et al. 2006, 2007; Gaidatzis et al. 2007). At a branch-length cutoff of 2.0, a large majority of these sites were in excess of the background (Fig. 2B, right). However, this high specificity came at a price, with many more sites detected above background at a less stringent cutoff of 1.0 (Fig. 2B).

Our more precise estimate of background conservation enabled robust detection of purifying selection for 6 mer seed matches that were not part of the larger, 7-8mer seed-matched sites (Fig. 2B). Ten thousand sites were conserved above background-a high number when considering the marginal efficacy of these 6 mer sites, as measured by monitoring mRNA destabilization or protein output after adding or disrupting miRNAs (Grimson et al. 2007; Nielsen et al. 2007; Baek et al. 2008; Selbach et al. 2008). Analysis of mRNA expression following ectopic addition of miRNAs into HeLa cells indicated that an offset 6 mer matching miRNA positions 3-8 (Fig. 1A) mediated mRNA destabilization approaching that of the seed-matched 6 mer, matching positions 2-7 (Fig. 2C), although the effects of the seed-match 6 mer were still significantly stronger ( $P=0.03$, two-sided KS test). This marginal yet detectable activity prompted us to explore the possibility that these offset 6 mer sites might also be selectively maintained. Our analysis, subtracting conservation due to 7- or 8-nt seed-matched sites as well as that attributed to matching seeds of related miRNAs, indicated that a small but detectable fraction of 
Friedman et al.

A

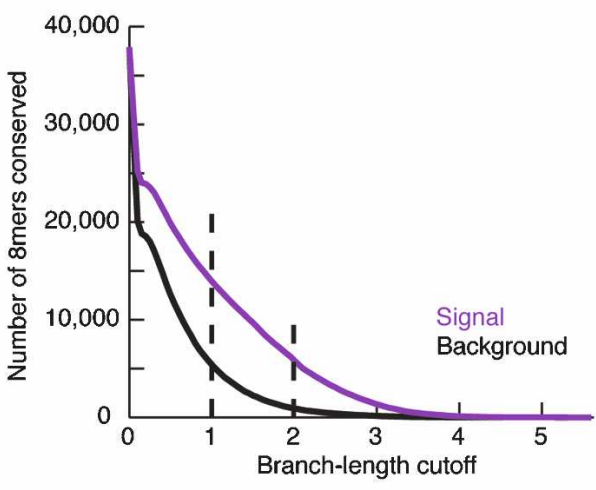

B

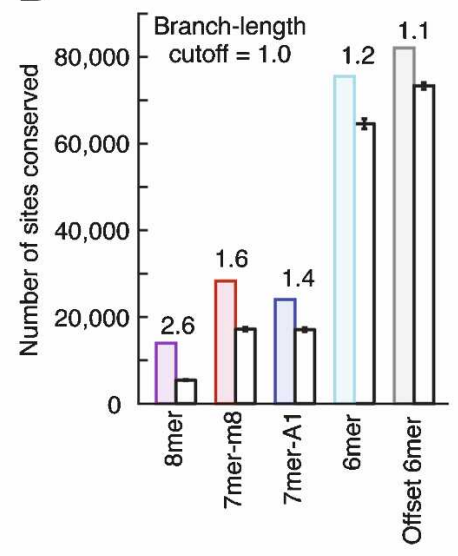

Branch-length

cutoff $=2.0$

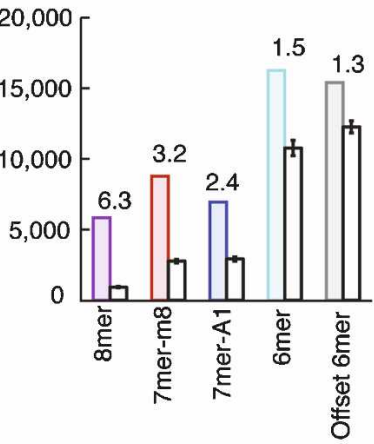

C

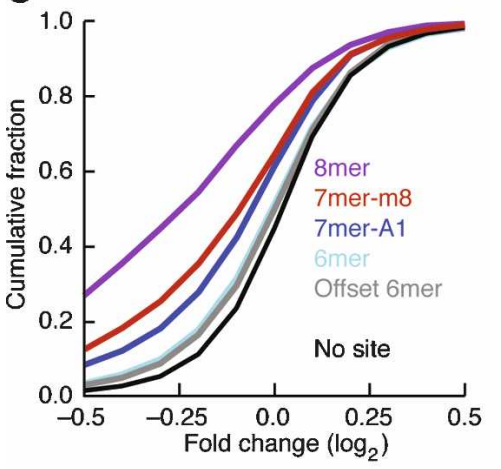

D

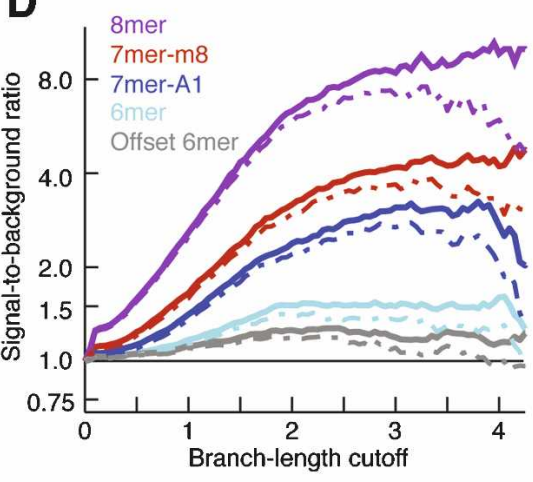

E

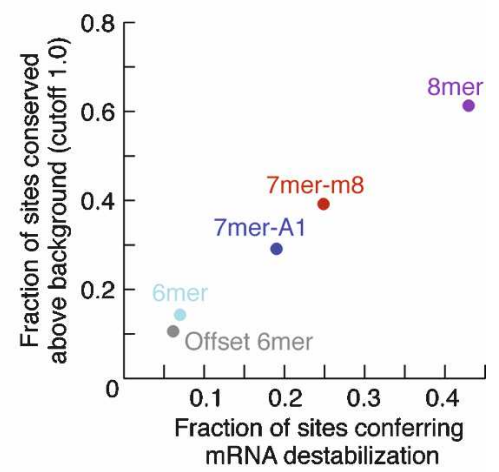

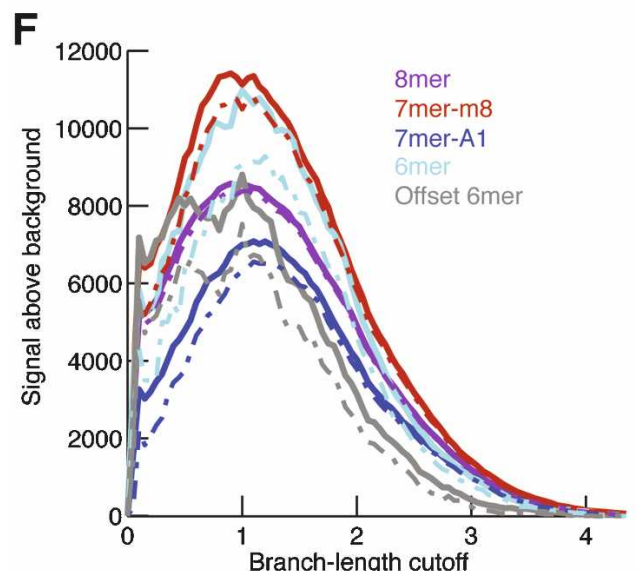

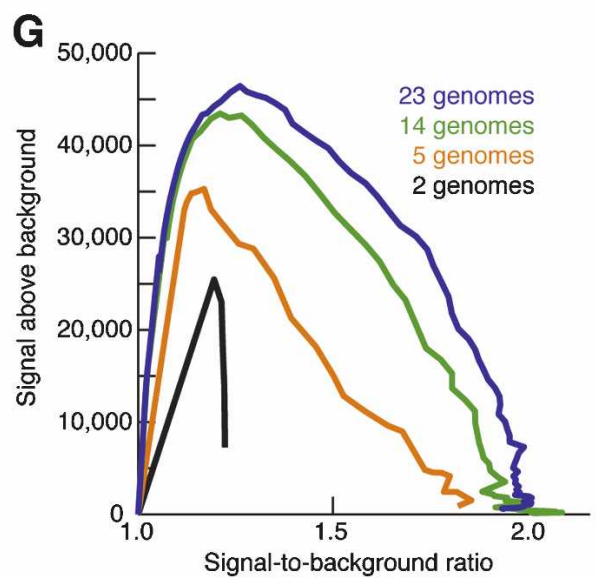

Figure 2. Conservation of major seed-match types. $(A)$ Conservation of $8 \mathrm{mer}$ sites for 87 broadly conserved miRNA families. High-sensitivity and high-specificity cutoffs are highlighted with broken lines at 1.0 and 2.0, respectively. (B) Conservation and background estimate for mutually exclusive site types at high sensitivity (left) and high specificity (right). The signal-to-background ratio is indicated above the pair of bars. Error bars indicate one standard deviation in the estimated background, based on subsampling of individual control k-mers. (C) Efficacy of offset 6mer sites. Microarray data monitoring mRNA destabilization following transfection of 11 miRNAs was analyzed as described previously (Grimson et al. 2007). Shown is the cumulative distribution of changes for transcripts containing exactly one offset $6 \mathrm{mer}$ site and no other canonical sites in their 3'UTR. For comparison, previously reported analyses of messages with single canonical sites are also shown (Grimson et al. 2007). (D) Signal-to-background ratio for indicated sites at increasing branch-length cutoff. Broken lines indicate 5\% lower confidence limit (z-test). (E) Correlation of site conservation rate and experimental efficacy. Fraction of sites conserved above background was calculated as ([Signal - Background]/Signal) at a branch-length cutoff of 1.0. The minimal fraction of sites conferring destabilization was determined from the cumulative distributions $(C)$, considering the maximal vertical displacement from the no-site distribution (correcting for the bumpiness of the distributions as described previously [Grimson et al. 2007]). ( $F$ ) Estimates of signal above background for the major site types. Broken lines indicate 5\% lower confidence limit ( $z$-test). (G) Aggregate conservation above background for all major site types when using using subsets of genomes. To facilitate overlay of the plots, the $X$-axis is signal-to-background ratio rather than branch-length cutoff. The 14-genome subset represents the non-fish species originally available in the UCSC 17-way alignments. The five-genome subset contains human, mouse, rat, dog, and chicken, and the two-genome subset contains only human and mouse.

\section{Genome Research}

www.genome.org 
these offset 6 mers were indeed selectively maintained (Fig. 2B). Because these 6 mer sites are so abundant in 3'UTRs, this small fraction corresponded to thousands of sites under purifying selection, which have been missed by algorithms that search for only seed-matched sites.

The result for the offset 6 mer raised the question of whether 6 mer matches to nearby miRNA segments might also be selectively maintained. Analysis of matches to miRNA segments 1-6, 4-9, and 5-10, excluding those sites that also possessed seed matches, revealed no 6 mer segments with appreciable signal above background (Supplemental Fig. 2). Parallel analyses of the mRNA expression data also failed to reveal 6 mer sites with efficacy approaching that of the 6 mer site corresponding to segment 3-8. We therefore focused on the selective conservation of the five types of sites that matched the seed region, one 8 mer, two 7 mers, and two 6 mers, which we refer to as the 6 mer and the offset 6 mer (Fig. 1A).

When examined over a broad range of branch-length cutoffs, signal-to-background ratios plateaued at a branch-length cutoff of about 3 (Fig. 2D), which exceeded the maximal branch length of the more highly conserved UTR bins. Larger signal-tobackground ratios implied higher fractions of seed matches under selection. For example, a signal-to-background ratio of 4.0 corresponds to $75 \%$ of matches being under purifying selection and thus presumably having conserved function. Regardless of the cutoff, the hierarchy of signal-to-background ratios remained constant, with 8 mer $>7$ mer-m $8>7$ mer-A $1>6$ mer $>$ offset $6 \mathrm{mer}$. Moreover, the signal-to-background ratio of the five site types, which indicated the fraction of sites under selection, corresponded well with the minimal fraction of sites conferring transcript destabilization following microRNA transfection, indicating a striking correlation between the selective maintenance of site types and their efficacy (Fig. 2E).

When considering the number of selectively maintained sites, a moderate branch-length cutoff of 1.0 yielded the highest signal above background (Fig. 2F). Increasing cutoffs from 1.0 to 2.0 yielded a tradeoff between increased specificity (Fig. 2D) and decreased sensitivity (Fig. 2F). For the five individual site types, the number of selectively maintained sites showed little correlation with the signal-to-background ratio. For example, the signalto-background ratio for the 6mer (1.2 at branch length 1.0) was far lower than that for the 8 mer (2.6 at branch length 1.0), but signal above background for the 6 mer (10,970 at branch length 1.0) was at least as high as that of the 8 mer (8543 at branch length 1.0). Thus, 3 'UTRs acquire and maintain marginally effective target sites in similar numbers as they do more highly effective sites. The 7 mer-m8 sites appear most important in terms of the number of sites under selection (Fig. 2F), whereas 8mers are the most important in terms of the proportion of sequences under selection and, equivalently, the power for prediction of individual targets (Fig. 2D).

Summing together the signal and background estimates for the five site types at the most sensitive conservation cutoff (1.0) yielded 46,441 \pm 2175 sites conserved above background (Fig. $2 \mathrm{~F})$, an average of $534 \pm 25$ per miRNA family (98 $\pm 2,128 \pm 7$, $80 \pm 8,126 \pm 22,101 \pm 14$ for 8 mer, 7 mer-m8, 7 mer-A1, 6mer, and offset 6 mer sites, respectively). This number of sites was nearly three times higher than the most sensitive previous estimate, which had required perfect 6 mer conservation in each of human, mouse, rat, and dog to detect 13,044 3'UTR sites conserved above background, or 210 sites conserved per miRNA family (Lewis et al. 2005). Several factors contributed to this large increase in the estimate of selectively maintained miRNA sites, including the improved methodology, larger and more accurate UTR and miRNA data sets, new genomes, and improved genome quality. To determine whether the principal factor was the newly available genomes, we performed the same analysis on subsets of genomes, keeping the UTR and miRNA data sets and methodology constant (Fig. 2G). The sensitivity was robust to the removal of a large number of genomes, suggesting that with current methods, the estimate of the number of selectively maintained sites will remain relatively constant with the addition of newly sequenced genomes.

Detection of selectively maintained sites with higher sensitivity implied that the number of conserved miRNA targets is far higher than previously estimated. Starting with all the sites detected at a given conservation cutoff and then randomly removing for each site type the number of sites corresponding to the predicted background in the relevant UTR bin yielded $9909 \pm 302$ genes targeted at a branch-length cutoff of 1.0. Using this method of sampling conserved sites, only $7 \%$ of genes had multiple conserved sites for the same miRNA family. Thus, for each miRNA family, the number of conserved targets $(497 \pm 49)$ approached the number of conserved sites $(534 \pm 25)$. Although more sites above background were predicted at the conservation cutoff of 1.0, the number of genes targeted reached a maximum of 10,739 \pm 564 at a branch-length cutoff of 0.6 , which corresponded to $57.8 \% \pm 3.0 \%$ of the human RefSeq data set. This percentage is about twice that of the most sensitive previous estimate (Lewis et al. 2005). Again, the number of targets per miRNA family $(438 \pm 60)$ approached the number of sites conserved above background per miRNA family (462 \pm 28$)$. Nonetheless, $72 \%$ of the 10,739 targeted messages had sites to multiple miRNA families, with an average of 4.2 sites per targeted 3'UTR. Indeed, the observed twofold increase in targeted UTRs from a threefold increase in site detection meant that our analysis added many additional newly predicted sites to previously predicted targets, thereby increasing not only the number of predicted targets but also the density of predicted targeting.

\section{Sites with seed bulges and mismatches are rarely under selection}

Having found a large and statistically significant number of conserved 6mer sites (Fig. 2F), despite their marginal efficacy (Fig. 2C), we investigated the possibility of selective conservation of imperfect seed matches, which also display severely compromised efficacy. Reasoning that if any mismatched sites were selectively maintained they would include those with the least disruptive mismatches, we focused on 8 mer matches containing either a single mismatch, a G:U wobble, a bulged nucleotide within the site, or a bulged nucleotide within the miRNA. In contrast to the canonical seed-matched types, these imperfect sites displayed little enrichment of conservation (Fig. 3A). For all four mismatched classes, signal-to-background ratio hovered near 1.0, rarely exceeding 1.1 at any branch-length cutoff, indicating that the number of sites under selection was at most a small fraction of the total (Fig. 3A). The 8mer with a bulge in the site was the only class for which the $5 \%$ confidence limit on the ratio consistently exceeded 1.0. This class of sites appeared to have a few hundred sites conserved above background, a number 10 times less than that of even the weakest seedmatched class (Fig. 3B). We cannot exclude the possibility that a very small fraction of other mismatched sites might also have been selectively maintained. However, because of the low signal- 

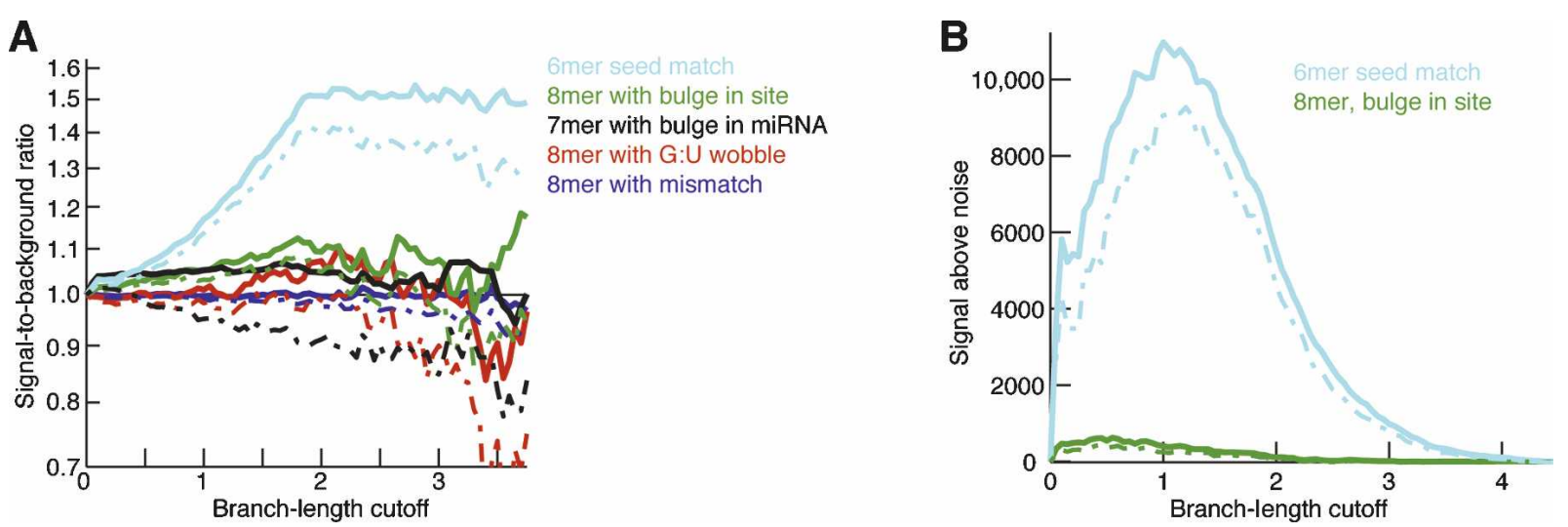

Figure 3. Occasional preferential conservation of imperfect sites. (A) Signal-to-background ratio for sites with the indicated single-nucleotide mismatches and bulges. A mismatch or G:U wobble must occur opposite miRNA seed nucleotides 2-7. A bulge in the site must occur between bases that pair to consecutive seed nucleotides 2-7, and a site creating a bulge must involve a 7 mer match that skips one of the seed nucleotides 2-7. Results for the canonical 6mer site (Fig. 2D) are included for comparison. Broken lines indicate 5\% lower confidence limit ( $z$-test). (B) Weak signal above background for a class of imperfect sites found to have a significantly positive signal-to-background ratio. Results for the canonical $6 \mathrm{mer} \mathrm{site} \mathrm{(Fig.} \mathrm{2F)}$ are included for comparison. Broken lines indicate 5\% lower confidence limit (z-test).

to-background ratio and low 5\% confidence estimate for the number of sites under selection, we conclude that seedmismatched sites are hardly ever selectively maintained and that including a substantial number of such sites when predicting targets would greatly compromise prediction specificity. These conclusions are supported by recent proteomic experiments demonstrating poor efficacy of targets predicted by methods that allow sites with seed mismatches (Baek et al. 2008; Selbach et al. 2008).

Selective maintenance of sites with a bulge in the site but not those with a bulge in the miRNA corresponds well with previous analyses of plant miRNA targeting (Mallory et al. 2004). This constraint observed in both plant and animal lineages can be explained by the idea that the Argonaute protein binds the miRNA backbone, preorganizing the miRNA seed region such that the Watson-Crick face is poised for pairing to the message (Bartel 2004). These contacts to the backbone in the seed region, presumably present before and after binding, would constrain the seed backbone, spacing each seed nucleotide such that a bulge in the miRNA would impose a gap in the site that would be difficult to span without disrupting adjacent pairs. In contrast, a bulged nucleotide in the site would be extruded into solvent and therefore more readily accommodated.

\section{Pairing to the $3^{\prime}$ end of miRNAs displays small but measurable excess conservation}

Although pairing to the $3^{\prime}$ region of the miRNA has long been thought to be consequential, evidence that such pairing enhances the efficacy of mammalian seed-matched sites has been obtained only recently (Grimson et al. 2007). Such sites in which 3 ' pairing productively augments seed pairing are called " 3 ' supplementary sites." Productive 3 ' pairing optimally centers on miRNA nucleotides 13-16 and the UTR region directly opposite this miRNA segment (Fig. 4A, top). Like seed pairing, 3' pairing appears relatively insensitive to predicted thermostability and instead quite sensitive to pairing geometry, preferring contiguous Watson-Crick pairs uninterrupted by bulges, mismatches, or G:U wobbles. These features are captured in a 3 '-pairing score, which awards one point for each contiguous Watson-Crick pair matching miRNA nucleotides 13-16 and a half point for each contigu- ous pair extending the pairing in either direction. Pairing segments offset from the miRNA are then penalized by subtracting a half point for each nucleotide of offset beyond \pm 2 nucleotides from the register directly opposite the miRNA, and then sites are assigned the score of the highest scoring pairing segment (Grimson et al. 2007). For example, the site shown in Figure 4A (top), which has seven contiguous, well-positioned pairs would be assigned a score of 5.5. Sites with scores $\geq 3$ display modestly increased efficacy and conservation (Grimson et al. 2007).

We set out to determine for each site type the selective maintenance of 3 '-supplementary pairing. At specified cutoffs for branch length and 3'-pairing score we determined the number of sites with supplementary $3^{\prime}$ pairing, estimating the background by repeating the analysis with a chimeric miRNA set created by swapping all possible $5^{\prime}$ and $3^{\prime}$ ends for miRNAs within our 87 miRNA families. For each site, the $3^{\prime}$ pairing score used was the maximum over all members of the miRNA family. For each of the four seed-matched types, and especially for the 7 mer-m8 site, selective maintenance of 3 '-supplementary pairing was confidently observed (Fig. 4A). As expected for a biological signal, specificity increased with greater conservation and with a greater 3 '-pairing score. Sensitivity peaked at a pairing score cutoff of 3.0, indicating that as few as 3-4 well-positioned supplementary pairs were selectively maintained (Fig. 4A). However, even at this sensitive cutoff, only $2281 \pm 537$ seed-matched sites had preferentially conserved $3^{\prime}$ pairing. Assuming that sites with selectively maintained $3^{\prime}$ pairing were also drawn from the pool of $\sim 44,000$ sites with selectively maintained matches to the seed region, we estimate that only $4.9 \% \pm 1.1 \%$ of all preferentially conserved sites have preferentially conserved 3 ' pairing. Nonetheless, for those rare sites with high $3^{\prime}$ pairing scores, consideration of supplemental pairing provided a useful boost to the overall signal-to-background ratio. For example, for the 498 mer sites with 3 '-pairing scores $\geq 5.0$ and branch-length values $\geq 2.0$, the aggregate signal-to-background ratio was estimated to be 13:1 (calculated as $6.3 \times 2.1$, using values from Figs. 2D and 4A, respectively), implying that the conservation of these individual sites was confidently attributed to miRNA targeting. For the remaining $95.1 \%$ of selectively maintained seed matches, which do not have preferential conservation of pairing to the $3^{\prime}$ end of miRNAs, the $3^{\prime}$ region of the miRNA might still in-

\section{Genome Research}

www.genome.org 
A
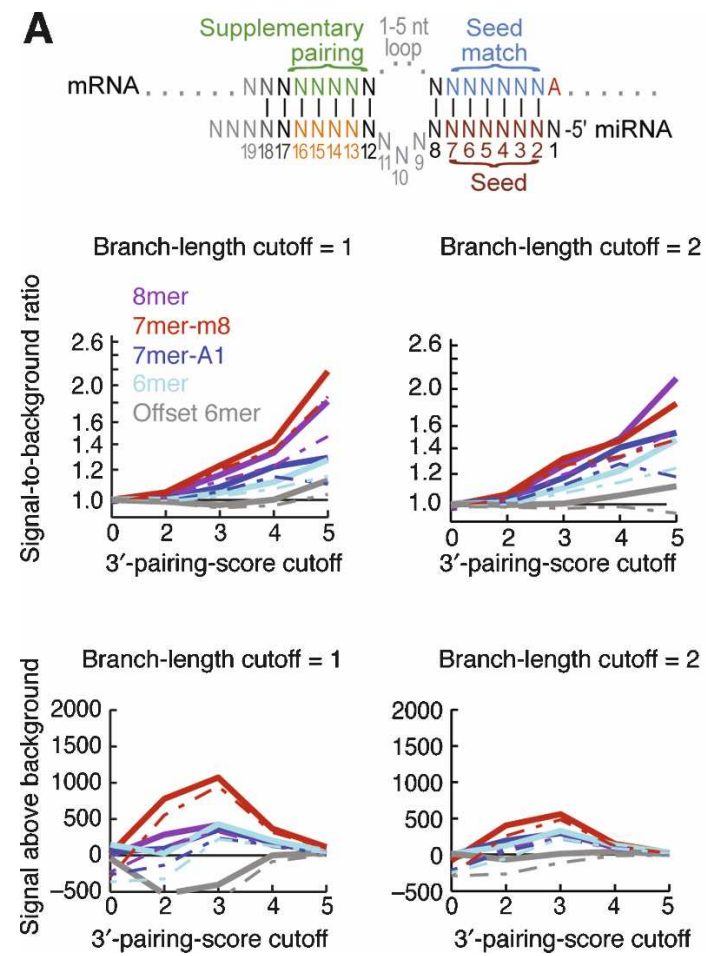
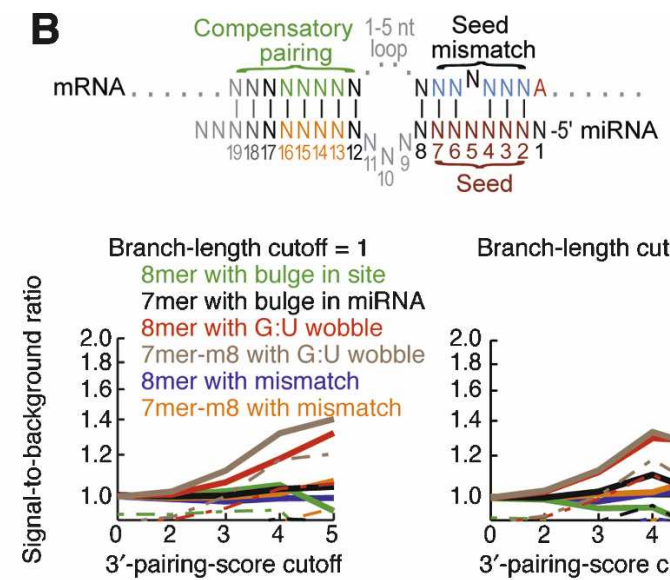

Branch-length cutoff $=2$
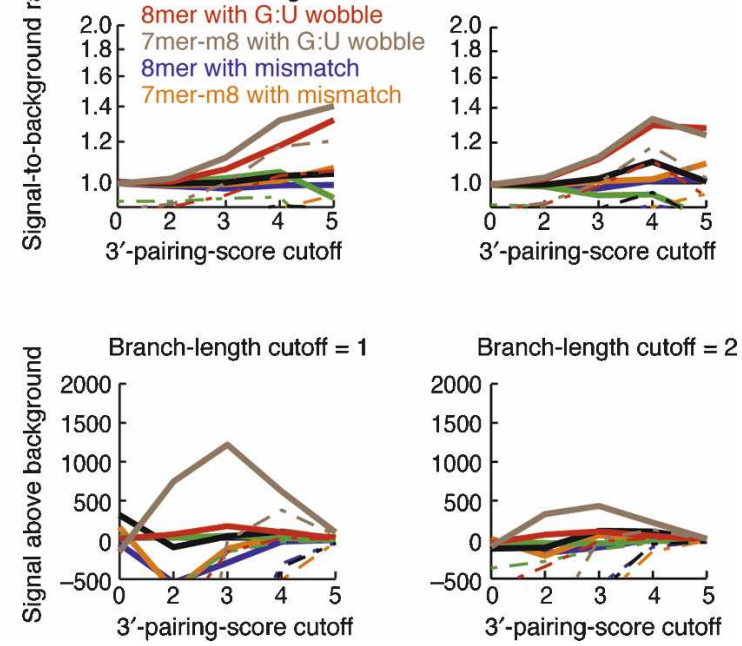

Figure 4. Conserved pairing to the $3^{\prime}$ ends of miRNAs. $(A)$ Preferential occurrence of pairing to the $3^{\prime}$ region of the miRNA, which can supplement canonical sites (diagrammed at top). Signal-to-background ratio (top two graphs) and signal above background (bottom two graphs) are plotted at conservation branch-length cutoffs of 1.0 (left) and 2.0 (right). Five percent confidence limits are shown as dashed lines. (B) Preferential occurrence of pairing to the $3^{\prime}$ region of the miRNA, which can compensate for mismatches or bulges in seed pairing (diagrammed at top). As in $A$, except that seed matches were replaced as indicated with sites containing a mismatched, G:U wobble, or bulged nucleotide.

teract with the message, but in a way that does not favor matches over mismatches and therefore does not add detectably to targeting specificity.

\section{Selective maintenance of 3 '-compensatory sites}

Pairing to the $3^{\prime}$ portion of the miRNA can not only supplement a 7-8mer match, it can also compensate for a single-nucleotide bulge or mismatch in the seed region, as illustrated by the let-7 miRNA sites in lin-41 and the miR-196 site in HOXB8 (Vella et al. 2004; Yekta et al. 2004). Such sites are called " 3 '-compensatory sites" (Fig. 4B, top). Previous analyses of mRNA array datafailed to detect efficacy of 3 '-compensatory sites, even when considering the principles that uncovered consequential $3^{\prime}$ supplementary pairing, which are embodied in the 3 '-pairing score (Grimson et al. 2007). This failure is attributed to the idea that 3 '-compensatory sites are exceedingly rare, presumably because the amount of pairing needed to compensate for seed mismatches is greater than that needed to supplement seedmatched sites, and as a result, a significant association is difficult to detect (Grimson et al. 2007). Supporting this idea, all experimentally validated examples of metazoan 3 '-compensatory sites involve pairing that centers on miRNA nucleotides 13-17 and extends to at least 9 contiguous Watson-Crick pairs, which corresponds to a 3 '-pairing score $\geq 6.5$ (Vella et al. 2004; Yekta et al. 2004).

An analysis of preferential site conservation could be more sensitive than that of array data, both because site-conservation analysis captures sites mediating translational repression without detectable mRNA destabilization and because site-conservation analysis can simultaneously consider many more miRNAs. With this possibility in mind, we examined the prevalence of $3^{\prime}$ compensatory sites, applying the same methodology as used for 3 '-supplementary sites. Sites with single-nucleotide mismatches or bulges had no enrichment in conserved or nonconserved $3^{\prime}$ pairing exceeding the $5 \%$ confidence threshold, regardless of the cutoffs (Fig. 4B). This result indicated that most of the bulged sites conserved above background (Fig. 3) are not conserved because they are 3 '-compensatory sites. However, 7-8mer sites with a single $G: U$ wobble had confidently enriched pairing at the relatively high 3 '-pairing-score cutoff of 4.0. At a branch-length cutoff of 1.0 , the number of sites within the $5 \%$ confidence interval numbered only 399 , an average of only 4.5 per miRNA family. Thus, our results support previous assertions that mismatched seed sites with 3 '-compensatory pairing are only rarely under selective pressure to be conserved (Brennecke et al. 2005; Lewis et al. 2005). Perhaps because such sites with extensive pairing to the $3^{\prime}$ portion of the miRNA possess much more informational complexity than do the 7-8mer perfect matches and therefore emerge much less frequently and are harder to maintain in evolution, these 3 '-compensatory sites appear to be used only rarely for biological targeting, comprising $1.5 \% \pm 0.7 \%$ of the conserved sites in mammals. The somewhat higher number of 3'-supplementary sites implies that $3^{\prime}$ pairing, when consequential, is principally a supplementary feature of canonical seed sites and more rarely plays a role in conferring activity to imperfect seed sites.

The paucity of 3 '-compensatory sites poses special challenges for confidently detecting conserved biological sites above 
Friedman et al.

background. Our use of the 3 '-pairing score and our observation that the G:U class of mismatches was more frequently compensated by conserved 3' pairing both represented important inroads into meeting this challenge. The 25 conserved $\mathrm{G}: \mathrm{U}$ sites with 3 '-pairing scores $\geq 6$ include the miR-196 site in the HOXB8 $3^{\prime}$ UTR, which has an off-scale $3^{\prime}$-pairing score of 9.0, and a similar miR-196 site in the HOXC8 3'UTR (Yekta et al. 2004). These 25 sites, together with the seven mismatched sites with scores $\geq 7$, are listed in Table 1 and will be included in the next release of TargetScan predictions (targetscan.org). Bulged sites with high 3 '-pairing scores $(\geq 6)$ did not appear preferentially conserved and thus are not included in the list.

\section{Mammalian-specific miRNAs have few selectively maintained} seed matches

An early study found that sites matching broadly conserved vertebrate miRNAs were more likely to be maintained than those matching mammalian-specific miRNAs (Lewis et al. 2003). Since then, target-prediction specificity has been estimated using only those miRNAs conserved to fish or chicken (Krek et al. 2005; Lewis et al. 2005; Gaidatzis et al. 2007), raising the question of whether the more recently emerged miRNAs have acquired enough conserved targets to detect any conservation signal above background. To address this question, we assembled a set of 53 miRNAs that were present in diverse placental mammals but absent in all sequenced chicken, lizard, and fish genomes (Supplemental Table 2). Examining the placental mammal subset of the phylog- eny, we found little preferential conservation for sites matching these mammalian-specific miRNAs (Fig. 5A). In contrast to sites matching broadly conserved miRNAs (Fig. 2D), the full 8mer was the only seed-match type with signal-to-background ratio consistently and confidently above 1.0 (Fig. 5A), and its ratio was no higher than that of offset 6 mers matching broadly conserved miRNAs.

The performance of the mammalian-only set also differed from that of the broadly conserved set when considering signal above background, with far fewer 8mers conserved above background (Fig. 5B). These differences could be due either to inherent differences in the miRNA sets, such as the level and breadth of expression, or to differential evolutionary time available for beneficial site emergence. To help differentiate between these possibilities, we screened matches to the broadly conserved miRNAs, removing all sites with seed matches conserved beyond mammals, thereby limiting the set of 8 mer sites to those more likely to have arisen in mammals after the divergence of mammals and other vertebrates. This removed more than half of the conserved sites matching the broadly conserved miRNAs, showing that part of the reason for the higher number of sites is the much greater time available for beneficial site emergence. However, even when considering the more restricted set of sites, and after normalizing for the numbers of miRNAs in the two sets, the broadly conserved miRNAs had more than four times as many selectively maintained 8mer matches per miRNA than did the mammalianspecific miRNAs (Fig. 5B), suggesting that the level and breadth of miRNA expression are also important factors. Combining the

Table 1. Conserved sites with imperfect seed pairing and high $3^{\prime}$-pairing scores

\begin{tabular}{|c|c|c|c|c|c|}
\hline 3'-Pairing score & Branch length & miRNA & RefSeq ID & Gene name & Seed type \\
\hline 9.0 & 1.85 & miR-196a & NM_024016 & HOXB8 & 8mer GU wobble \\
\hline 7.5 & 1.2 & miR-145 & NM_030809 & FAM130A1 & 8 mer mismatch \\
\hline 7.0 & 3.25 & miR-365 & NM_002398 & MEIS1 & 8mer mismatch \\
\hline 7.0 & 2.6 & miR-519d & NM_153020 & RBM24 & 8 mer mismatch \\
\hline 7.0 & 1.85 & miR-153 & NM_032521 & PARD $6 B$ & 7mer mismatch \\
\hline 7.0 & 1.6 & miR-590-5p & NM_033656 & BRWD1 & 7mer mismatch \\
\hline 7.0 & 1.35 & miR-29b & NM_024834 & C10orf119 & 7mer mismatch \\
\hline 7.0 & 1.15 & miR-222 & NM_002855 & PVRL1 & 8 mer mismatch \\
\hline 6.5 & 1.7 & miR-19b & NM_017637 & $B N C 2$ & 8mer GU wobble \\
\hline 6.5 & 1.5 & miR-613 & NM_014903 & NAV3 & 7mer GU wobble \\
\hline 6.5 & 1.35 & miR-191 & NM_134265 & WSB1 & 7mer GU wobble \\
\hline 6.5 & 1.3 & miR-15b & NM_001039590 & USP9X & 7mer GU wobble \\
\hline 6.5 & 1.15 & miR-145 & NM_001039457 & ATP6VOB & 7mer GU wobble \\
\hline 6.0 & 3.5 & miR-301a & NM_022893 & $B C L 11 A$ & 7mer GU wobble \\
\hline 6.0 & 3.45 & miR-196a & NM_022658 & HOXC8 & 8mer GU wobble \\
\hline 6.0 & 3.4 & miR-19a & NM_016396 & CTDSPL2 & 7mer GU wobble \\
\hline 6.0 & 2.95 & miR-20a & NM_015215 & CAMTA1 & 8mer GU wobble \\
\hline 6.0 & 2.55 & miR-130a & NM_004721 & MAP $3 K 13$ & 7mer GU wobble \\
\hline 6.0 & 2.15 & miR-106b & NM_020814 & MARCH4 & 7mer GU wobble \\
\hline 6.0 & 2.15 & miR-424 & NM_001418 & EIF4G2 & 8 mer GU wobble \\
\hline 6.0 & 2.1 & $\mathrm{miR}-302 \mathrm{~d}$ & NM_002024 & FMR1 & 7mer GU wobble \\
\hline 6.0 & 1.8 & miR-520e & NM_014494 & $T N R C 6 A$ & 7mer GU wobble \\
\hline 6.0 & 1.75 & miR-190 & NM_001003652 & SMAD2 & 8mer GU wobble \\
\hline 6.0 & 1.55 & miR-424 & NM_007374 & $5 I X 6$ & 7mer GU wobble \\
\hline 6.0 & 1.3 & miR-130a & NM_012308 & $F B X L 11$ & 7mer GU wobble \\
\hline 6.0 & 1.25 & miR-519d & NM_005808 & CTDSPL & 8mer GU wobble \\
\hline 6.0 & 1.2 & miR-190 & NM_201572 & CACNB2 & 8mer GU wobble \\
\hline 6.0 & 1.2 & miR-519d & NM_001012393 & OPCML & 7mer GU wobble \\
\hline 6.0 & 1.15 & miR-15b & NM_152277 & UBTD2 & 7mer GU wobble \\
\hline 6.0 & 1.1 & miR-129-5p & NM_020801 & $A R R D C 3$ & 7mer GU wobble \\
\hline 6.0 & 1.1 & miR-33a & NM_178826 & ANO4 & 7mer GU wobble \\
\hline 6.0 & 1.05 & miR-302c & NM_015215 & CAMTA1 & 7mer GU wobble \\
\hline
\end{tabular}

Listed are all $7 \mathrm{mer}$ and $8 \mathrm{mer} \mathrm{G}: \mathrm{U}$ wobble sites with $3^{\prime}$-pairing score $\geq 6.0$ and branch length $\geq 1.0$. Also listed are all $7 \mathrm{mer}$ and $8 \mathrm{mer}$ mismatched sites that meet the above criteria and have human $3^{\prime}$-pairing score $\geq 7.0$. 
A

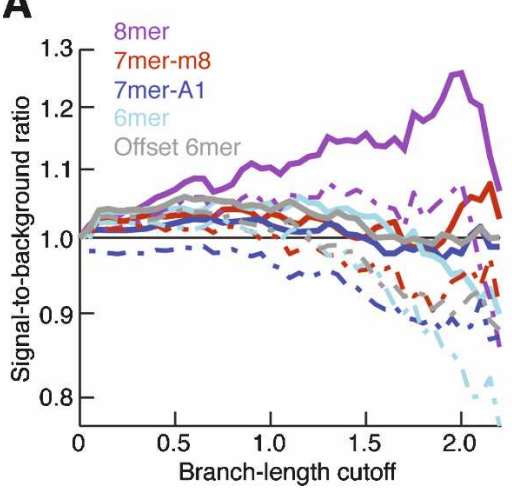

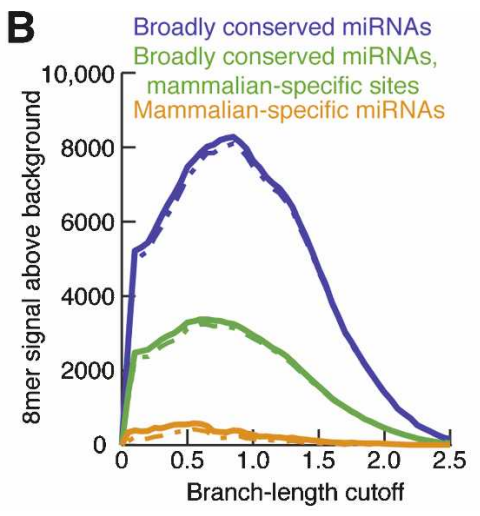

Figure 5. Conservation of sites matching mammalian-specific miRNAs. ( $A$ ) Signal-to-background ratio for sites matching 53 mammalian-specific miRNA families in 18 placental mammals, otherwise as in Figure 2D. (B) Signal above background for 8mer sites matching either broadly conserved or mammalian-specific miRNAs in 18 placental mammals, otherwise as in Figure 2F. Analysis of 8 mer sites matching broadly conserved miRNAs considers either all sites (blue) or excludes those sites conserved beyond placental mammals (green). (C) Signal-to-background ratios for 8 mer sites matching individual miRNAs in orthologous 3'UTRs of placental mammals at optimal sensitivity (branch-length cutoff of 0.85 ). For the broadly conserved miRNA set, conservation signal excludes sites conserved beyond placental mammals. Distributions expected if miRNA targeting conferred no preferential conservation were estimated using the average signal-to-background ratio of $8 \mathrm{mer}$ controls selected for each site, considering GC content and dinucleotide-based conservation (broken lines). Expectations differed between the two sets because of different miRNA numbers and different dinucleotide compositions.

signal above background for all site types, the differential appeared far greater, with the mammalian miRNAs averaging only $10.9 \pm 3.0$ selectively maintained sites.

To determine whether a few of the mammalian-only miRNAs might have conservation patterns resembling those of the broadly conserved set, we examined the conservation of $8 \mathrm{mer}$ seed matches corresponding to individual miRNAs. As expected, the signal-to-background ratios of the broadly conserved miRNAs fell mostly outside the control distribution (Fig. 5C). The observation that most fell outside the control distribution illustrated that the high signals observed for these broadly conserved miRNAs in aggregate also applied to most of them individually and could not be attributed to chance overlap of a few seed matches to a few highly conserved regulatory sequences. In contrast, the mammalian-specific miRNAs had a distribution only slightly shifted from that of the controls, with an excess of -5-10 miRNAs in the 1.5-2.5 signal-to-background range. We conclude that only a small subset of mammalian-specific miRNAs (including most prominently miR-487b, miR-127-3p, miR379, and miR-543; Supplemental Table 2) have a measurable number of selectively maintained sites and caution that for the majority of mammalian-specific miRNAs, the observation that a site is conserved provides no evidence that it has biological function.

\section{Estimating confidence for selective maintenance of individual sites}

The widespread scope of conserved targeting brings to the fore the question of which of these many miRNA target interactions can be predicted with confidence. One raw indicator is the conservation branch length of the site-clearly, more highly conserved sites are more likely to be under selection, particularly when controlling for differential UTR conservation, as in our method. However, our branch-length values did not account for the type of site and its sequence features. For example, a branch length of 1.0 for an 8mer is far more compelling evidence for selective maintenance than a branch length of 3.0 for an offset $6 \mathrm{mer}$ (Fig. 2D). To control for site type and sequence features, we used our previously described controls to calculate a signal-to-background ratio (S/B) for each site at each branch length. For the purpose of evaluating individual sites, assessing controls at each branch length instead of at each branch-length cutoff is necessary to avoid crediting poorly conserved sites for having the same sequence as many highly conserved sites. We then converted this S/B to a probability of conserved targeting $\left(P_{\mathrm{CT}}\right)$, which is approximately equal to $(\mathrm{S} / \mathrm{B}-1) /(\mathrm{S} / \mathrm{B})$ (or near zero, for sites with $\mathrm{S} / \mathrm{B}<1)$. This score reflected the Bayesian estimate of the probability that a site is conserved due to selective maintenance of miRNA targeting rather than by chance or any other reason not pertinent to miRNA targeting, allowing for uncertainty in the S/B ratio. For predicting biologically conserved target sites, the score represented 1 -FDR, where FDR was our estimate of the falsediscovery rate. This deceptively simple value incorporated knowledge of the conservation level of a particular site, the seedmatch type, the number of selectively maintained seed matches for the particular miRNA, the background conservation level for the $k$-mer, and the UTR conservation context.

The $P_{\mathrm{CT}}$ provides a useful criterion for assessing the biological relevance of predicted miRNA-target interactions. Selectively maintained sites are more likely to have detectable biological function, are more likely to have functions in experimental animals that are pertinent to humans, and tend to be more effective (Grimson et al. 2007; Nielsen et al. 2007). To illustrate the ability of this measure to predict site efficacy, we turned to experimental data examining mRNA destabilization after introducing miRNAs (Grimson et al. 2007). As expected, site $P_{\mathrm{CT}}$ correlated with the mean level of mRNA destabilization (Fig. 6A). In addition, we tested a subset of miRNA sites that were previously considered nonconserved because they were not found in mouse, rat, or dog alignments (Lewis et al. 2005). Those sites newly recognized as selectively maintained were substantially more responsive to the transfected miRNAs than were those still lacking a signal for conserved targeting (Fig. 6B).

Having established a more sensitive and precise tool for evaluating site conservation, we overhauled the TargetScan web resource, separating conserved and highly conserved 3'UTR targets of each miRNA based on the $P_{\mathrm{CT}}$ values of their $7-8$ mer sites, 

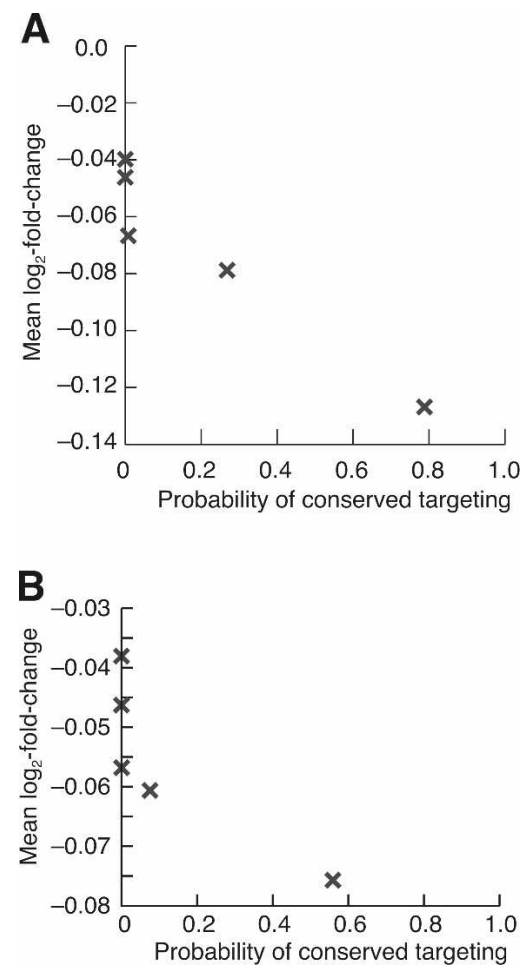

Figure 6. Correlation of $P_{\mathrm{CT}}$ with mRNA destabilization. $(A)$ Destabilization of human messages with exactly one 7 mer-m8 3'UTR site to a transfected miRNA (Grimson et al. 2007). Messages were grouped into six equal bins based on the site $P_{\mathrm{CT}}$. (B) Destabilization of human messages with exactly one $7 \mathrm{mer}-\mathrm{m} 8 \mathrm{3}^{\prime} \mathrm{UTR}$ site to a transfected miRNA (Grimson et al. 2007), considering only those sites that were not conserved in either mouse, rat, or dog.

compiling an aggregate probability of conserved targeting $\left(\mathrm{a} P_{\mathrm{CT}}\right)$ for those targets with multiple sites for that miRNA, calculated as $\mathrm{a} P_{\mathrm{CT}}=1-\left(\mathrm{FDR}_{\text {site } 1} \times \mathrm{FDR}_{\text {site } 2} \times \mathrm{FDR}_{\text {site } 3} \ldots\right)$. To capture sites that were missing in the annotated human $3^{\prime}$ UTRs but present in the mouse annotations, a mouse-centric version of the analyses was performed in parallel. This improved web resource, called TargetScan version 5.0, also lists the $P_{\mathrm{CT}}$ for each 7-8mer site, with the option of sorting the predicted targets of each miRNA by their $\mathrm{a}_{\mathrm{CT}}$. Retained in TargetScan 5.0 are site context scores, which consider features such as the AU content in the vicinity of the site and the position of the site within the message, to predict the function and quantitative efficacy of each site (Grimson et al. 2007). Because the context scores are determined based on information completely orthogonal to site conservation, the $P_{\mathrm{CT}}$ values and the context scores provide independent and complementary information useful for predicting the biological relevance and efficacy of each site.

\section{The widespread scope of conserved targeting}

One of the key results of applying our new methods for quantitatively evaluating site conservation to the study of miRNA targeting was the sheer number of preferentially conserved sites. When considering only the 6-8mer perfect $3^{\prime}$ UTR matches to the seed regions of broadly conserved miRNAs, 46,441 \pm 2175 sites were conserved above background, implying that $57.8 \% \pm 3.0 \%$ of the human genes are conserved miRNA targets. Considering also imperfectly matched sites added another $625 \pm 239$ preferentially conserved sites to the total (Fig. 3B); 3'-compensatory sites added another $714 \pm 315$ (Fig. 4B), sites matching more narrowly conserved miRNAs added another $578 \pm 158$ (Fig. 5B), altogether modestly raising the estimated number of preferentially conserved $3^{\prime}$ UTR sites to 48,360 . Not considered are the human miRNAs that have escaped confident annotation. However, because these miRNAs have remained unannotated, in part because they are more narrowly conserved and have more restrictive expression domains, our results for the mammalian-specific miRNAs suggest that eventual consideration of these unannotated miRNAs will add only modestly to the current picture of conserved targeting. We suspect that a more significant increase will come by considering targeting in ORFs, which is thought to be widespread, although less than that in 3'UTRs (Lewis et al. 2005; Stark et al. 2007b). Further increases will come with improved UTR annotations and the consideration of alternative 3'UTRs. Anticipating these additional sites, we can say with confidence that over $60 \%$ of human protein-coding genes are conserved targets of miRNAs, with our best estimate of the actual fraction of human protein-coding genes under pressure to maintain pairing to miRNAs falling above this, at about two thirds of all protein-coding genes.

One surprise of our analysis was the substantial number of selectively maintained $6 \mathrm{mer}$ and offset 6 mer sites, numbering $10,970 \pm 1909$ and $8803 \pm 1276$, respectively. Indeed, when excluding these sites and considering only the 7-8mer sites, our estimate of the number of conserved targets per broadly conserved miRNA dropped to $292 \pm 18$, which corresponded to $44.5 \pm 3.4 \%$ of the RefSeq genes. With the exception of an offset 6 mer site for let-7 miRNA in the human LIN28 3'UTR (Wu and Belasco 2005), 6mer sites typically have very poor efficacy when examined experimentally, as if the majority are inert, and nearly all the rest have very marginal influence on protein output (Fig. 2C; Grimson et al. 2007; Nielsen et al. 2007; Baek et al. 2008; Selbach et al. 2008). Yet 3'UTRs selectively maintain these 6 mer target sites in similar numbers as they do 7 and 8 mer sites. Of course, 6 mers are much easier to access by mutation and then preserve from mutation, but what would be the selection pressure to preserve such minor effects? One conundrum in the field of miRNA-mediated gene regulation is why so many 7-8mer sites would be conserved so broadly in metazoan 3'UTRs, when each down-regulates protein output by very little-nearly always less than $50 \%$ and usually less than $30 \%$, which would only very rarely produce observable phenotypic consequences (Baek et al. 2008). But the 7-8mer conundrum pales when considering the selective maintenance of 6 mers, which appear to tweak gene expression so finely that the effects are difficult to detect at the molecular level, let alone the phenotypic level.

One way to reconcile the high number of preferentially conserved 6-nt sites with the modest efficacy of these sites is to propose that these conserved 6mers are not preferentially conserved based on their activity as 6-nt sites, but instead, represent the inactive (or less active) decay products of preferentially conserved 7-8mer sites. When considering this explanation, two scenarios could contribute the conserved 6 mer signals. In one scenario, the extended site has degraded to one of the 6mers in the human lineage. For example, a 7 mer site that functions in most animals but has decayed to an inactive 6 mer in primates would have contributed to our count of conserved 6 mers in human UTRs. In the second scenario, the extended site is conserved in the UTR of human and other species but exceeds the branch-length cutoff

\section{Genome Research}

www.genome.org 
only when including additional species in which it has degraded to one of the 6mers. For example, if the 8mer site conserved in the SPRED1 UTR had degraded to an inactive 6mer in hedgehog, then the site would have contributed to our count of conserved 8 mers at branch-length cutoff of 0.9, but not at 1.0; at a cutoff of 1.0 it would have contributed to our count of conserved 6 mers. When performing the analyses so as to exclude these two scenarios, our estimate of the number of preferentially conserved sites per highly conserved miRNA family dropped $35 \%$, to an average of $77 \pm 22$ for 6 mer sites and $69 \pm 15$ for offset 6 mer sites (Supplemental Discussion). Thus, some of the preferential conservation of 6-nt matches to the seed region can be explained by their relationship with conserved 7 mers, but thousands of 6 mers and offset 6 mers are selectively maintained without the aid of 7 mer conservation. Moreover, because these decreases in preferentially conserved 6-nt sites imply corresponding increases in preferentially conserved 7-8mer sites, consideration of these scenarios does not change our estimates of the total number of preferentially conserved sites and the total number of conserved mammalian targets.

Our observation that these 6 mer sites have been selectively maintained so frequently implies the existence of widespread pressure for finely adjusted protein output with surprisingly narrow tolerances for optimal expression in different cell types. The observation that this selective pressure persists over such long branch lengths indicates that these optimal expression levels and narrow tolerances persist in the face of many other changes over surprisingly long evolutionary distances. These results become somewhat easier to reconcile when considering the possibility that 6mers might impart more robust repression in particular cells or conditions that have yet to be accessed experimentally. Hinting at this possibility is the more readily detected activity of 6 mers matching miR-430 during the maternal-to-zygotic transition, a developmental stage at which the miR-430 family comprises most of the miRNA molecules in the animal (Giraldez et al. 2006). An alternative possibility is that the 6 mer and offset $6 \mathrm{mer}$ impart some function other than repressing protein output. For example, if transient miRNA association played a widespread role in mRNA subcellular localization, then many 6 mer sites could be conserved without imparting any repression. In either scenariowidespread and persistently narrow gene-expression tolerances or expanded miRNA function-the discovery that so many 6 mers and offset 6 mers are selectively maintained sets the stage for important future insights into miRNA biology.

\section{Methods}

\section{Alignments and phylogeny}

Sequences (3'UTR) were defined as the longest $3^{\prime}$ UTR for each human RefSeq gene (Pruitt et al. 2005), resulting in 18,577 unique UTRs for 18,577 genes. Human UTR boundaries were used to acquire orthologous UTRs from the 28-genome vertebrate sequence alignments from the UCSC genome browser (Karolchik et al. 2008). The average phylogeny was determined using the branching structure provided by UCSC, and branch lengths were estimated by running DNAML (part of the PHYLIP package [Felsenstein 1989]) on the UTR alignments. To define UTR conservation bins, human UTRs were ranked using the median branch length over single bases. Then, DNAML was run on the sequences in each UTR bin, creating phylogenies with the same branching structure but modified branch lengths scaled for each UTR bin.

\section{miRNA sequences}

The broadly conserved miRNA set comprised all 79 families of miRBase entries (release 10.0) with both a human and zebrafish miRNA sharing the same seed sequence (Supplemental Table 1). Additionally, we included 8 human miRNA families with mouse miRBase entries and a conserved foldback in lizard, chicken, frog, or another fish genome, yielding a total of 87 miRNA families. To generate the mammalian-specific miRNA set, miRbase entries for human miRNAs conserved to mouse were manually inspected for aligned sequences from most of the sequenced mammals, perfect conservation of the seed sequence within placental mammals that had aligned sequence, no conservation in species other than placental mammals, and good predicted folding characteristics in most placental mammals. Seven of the remaining miRNAs whose seed matches had strong excess conservation ( $>2.0 \mathrm{~S} / \mathrm{B}$ ratio) in species other than placental mammals were also removed (reasoning that this excess conservation was likely caused by an miRNA ortholog that escaped detection because of imperfect alignments or sequencing coverage), leaving 53 mammalianspecific miRNAs (Supplemental Table 2). A list of miRNAs left out of the mammalian-specific category for various reasons is found in Supplemental Table 3.

\section{Background estimation}

For each seed-match $k$-mer, possible control $k$-mers were first filtered to remove seed matches to other miRNAs, and to have the same length, number of $\mathrm{G}+\mathrm{C}$ bases, and possible matches to PUF proteins (UGU[A/G]) as the seed match. In the case of 8 mer and 7 mer-A1 matches, the controls were also constrained to have an $\mathrm{A}$ in the 3 '-most nucleotide. For each branch-length cutoff, the 50 control $k$-mers with the closest expected conservation rate to the seed-match $k$-mer were selected. Expected conservation was calculated using a first-order Markov model with parameters derived from the empirical dinucleotide conservation rate in $3^{\prime}$ UTRs at a particular branch-length cutoff. In other words, control $k$-mers were picked to exactly match length, GC content, and PUF binding sites, and to closely match the expected conservation rate based on the dinucleotide content. The background estimate was the number of occurrences of the $k$-mer multiplied by the average fraction of sites conserved in control $k$-mers at the same branch length. As done for the signal, background was determined for each UTR bin separately, and then at each branch length, background from the 10 UTR bins was summed to generate the overall background distribution. At each branch length, confidence intervals were calculated using the Gaussian distribution with mean equal to the background estimate and standard deviation calculated using the background estimates given by individual control $k$-mers as opposed to the average over all 50.

\section{Seed-match types}

For the five major seed-match types (8mer, 7 mer-m8, 7mer-A1, $6 \mathrm{mer}$, offset $6 \mathrm{mer}$ ), raw signal and background values were calculated without regard to whether a shorter seed match was nested within a longer one. Then, for each miRNA and for each branchlength cutoff, the signal for the 8mer match was subtracted from the signal of the corresponding 7 mer-m 8 match, and the $8 \mathrm{mer}$ background estimate was subtracted from the $7 \mathrm{mer}-\mathrm{m} 8$ estimate. Similarly, 8mer signal and background were subtracted from signal and background of the other seed-match types, 7 mer-m8 signal and background were subtracted from that of both 6 mers and offset 6 mers, and 7 mer-A 1 signal and background was subtracted from that of 6 mers. In cases in which a particular seed match could be considered a $7 \mathrm{mer}-\mathrm{m} 8$ match to one miRNA and a $7 \mathrm{mer}-$ 
A1 to another miRNA, it was considered only as a 7 mer-m8 match to avoid double-counting. Likewise, seed matches ambiguous between 6 mers and offset 6 mers were considered as only 6mers. Seed matches with mismatches, G: $U$ wobbles, or bulges were filtered so that they did not contain perfect seed matches to other miRNAs.

\section{3' pairing}

Pairing scores (3') were calculated as previously described (Grimson et al. 2007). For sites matching miRNA families comprising miRNAs with different 3 ' sequences, the 3 '-pairing score used was the maximum score for any member of the miRNA family. The branch length of a site at a particular 3'-pairing score was calculated for the set of species having both the seed match conserved and a 3'-pairing score at least that of the human score. Control 3 '-pairing scores and conservation were estimated by swapping every miRNA seed and 3 '-end family, and recalculating 3 '-pairing scores for every combination. Background estimate and confidence intervals were calculated as before, except that there were 86 control 3 '-end families for each swap instead of 50 control $k$-mers. For compensatory pairing sites in Table 1 , orthologous sites were considered conserved if their 3'-pairing score was greater than 6.0, regardless of the human pairing score. This was done to capture well-conserved human sites that have recently extended already strong 3 ' pairing.

\section{Probability of conserved targeting}

For each human seed-match site, the conservation signal and background were calculated using methods analogous to those described above. Rather than using a branch-length cutoff, we used a branch-length window with a size set to meet a minimum of 20 occurrences of the site in human UTRs and calculated the fraction of seed matches conserved within the branch-length window. In the few cases with less than 20 total occurrences of the seed match, all corresponding sites were assigned a $P_{\mathrm{CT}}$ of 0 . For the remaining sites, the $P_{\mathrm{CT}}$ was defined as $\mathrm{E}[(\mathrm{S}-\mathrm{B}) / \mathrm{S}]$ where $\mathrm{B}$, the background estimate, is a constant, and $\mathrm{S}$ is a random variable. $S$ is defined as $\max \left\{S_{\text {obs }} / \mathrm{N}, \mathrm{B}\right\}$, where $\mathrm{S}_{\text {obs }}$ is the observed signal and $\mathrm{N}$ is the total number of occurrences, distributed around the observed total $\mathrm{N}_{\mathrm{obs}}$ as Poisson $\left(\mathrm{N}_{\mathrm{obs}}\right)$. Because the background estimate is based on a mean of many measurements, but the signal is based on a single observation, we fix the number of conserved occurrences $\left(\mathrm{S}_{\mathrm{obs}}\right)$ and allow the total number of occurrences $(\mathrm{N})$ to vary. Thus, the $P_{\mathrm{CT}}$, which ranges between 0 and 1, corresponds to a Bayesian estimate of the probability that a site conserved to a particular branch length is conserved due to miRNA targeting. For some miRNAs, we observed high variability of $P_{\mathrm{CT}}$ values for sites with close branch-length values; therefore, we implemented a smoothing procedure when deriving $P_{\mathrm{CT}}$ values reported at the TargetScan site (Supplemental Discussion).

\section{Acknowledgments}

We thank members of the Bartel and Burge laboratories for helpful discussions, and the Broad Institute of MIT and Harvard, the Human Genome Sequencing Center at the Baylor College of Medicine, the Genome Sequencing Center at Washington University, and the Department of Energy's (DOEs) Joint Genome Institute for providing access to unpublished vertebrate genome sequences. We also thank George Bell, Katherine Gurdziel, Prathapan Thiru, Bingbing Yuan, and Fran Lewitter for implementing our improved methods at the TargetScan website. This work was supported by a Krell Institute/Department of Energy
Computational Sciences Graduate Fellowship (to R.C.F.) and by grants from the NIH to C.B.B. and D.P.B.

\section{References}

Baek, D., Villen, J., Shin, C., Camargo, F.D., Gygi, S.P., and Bartel, D.P. 2008. The impact of microRNAs on protein output. Nature 455: 64-71.

Bartel, D.P. 2004. MicroRNAs: Genomics, biogenesis, mechanism, and function. Cell 116: 281-297.

Blanchette, M., Kent, W.J., Riemer, C., Elnitski, L., Smit, A.F., Roskin, K.M., Baertsch, R., Rosenbloom, K., Clawson, H., Green, E.D., et al. 2004. Aligning multiple genomic sequences with the threaded blockset aligner. Genome Res. 14: 708-715.

Brennecke, J., Stark, A., Russell, R.B., and Cohen, S.M. 2005. Principles of microRNA-target recognition. PLoS Biol. 3: e85. doi: 10.1371/journal.pbio.0030085.

Farh, K.K., Grimson, A., Jan, C., Lewis, B.P., Johnston, W.K., Lim, L.P., Burge, C.B., and Bartel, D.P. 2005. The widespread impact of mammalian microRNAs on mRNA repression and evolution. Science 310: $1817-1821$.

Felsenstein, J. 1989. PHYLIP-phylogeny inference package (version 3.2). Cladistics 5: 164-166.

Gaidatzis, D., van Nimwegen, E., Hausser, J., and Zavolan, M. 2007. Inference of miRNA targets using evolutionary conservation and pathway analysis. BMC Bioinformatics 8: 69 . doi: 10.1186/1471-2105-8-69.

Giraldez, A.J., Mishima, Y., Rihel, J., Grocock, R.J., Van Dongen, S., Inoue, K., Enright, A.J., and Schier, A.F. 2006. Zebrafish MiR-430 promotes deadenylation and clearance of maternal mRNAs. Science 312: $75-79$.

Grimson, A., Farh, K.K., Johnston, W.K., Garrett-Engele, P., Lim, L.P., and Bartel, D.P. 2007. MicroRNA targeting specificity in mammals: Determinants beyond seed pairing. Mol. Cell 27: 91-105.

Grun, D., Wang, Y.L., Langenberger, D., Gunsalus, K.C., and Rajewsky, N. 2005. microRNA target predictions across seven Drosophila species and comparison to mammalian targets. PLoS Comput. Biol. 1: e13. doi: 10.1371/journal.pcbi.0010013.

Hwang, D.G. and Green, P. 2004. Bayesian Markov chain Monte Carlo sequence analysis reveals varying neutral substitution patterns in mammalian evolution. Proc. Natl. Acad. Sci. 101: 13994-14001.

Karolchik, D., Kuhn, R.M., Baertsch, R., Barber, G.P., Clawson, H., Diekhans, M., Giardine, B., Harte, R.A., Hinrichs, A.S., Hsu, F., et al 2008. The UCSC Genome Browser Database: 2008 update. Nucleic Acids Res. 36: D773-D779. doi: 10.1093/nar/gkm966.

Kauppi, L., Jeffreys, A.J., and Keeney, S. 2004. Where the crossovers are: Recombination distributions in mammals. Nat. Rev. Genet. 5: 413-424.

Kheradpour, P., Stark, A., Roy, S., and Kellis, M. 2007. Reliable prediction of regulator targets using 12 Drosophila genomes. Genome Res. 17: 1919-1931.

Krek, A., Grun, D., Poy, M.N., Wolf, R., Rosenberg, L., Epstein, E.J., MacMenamin, P., da Piedade, I., Gunsalus, K.C., Stoffel, M., et al. 2005. Combinatorial microRNA target predictions. Nat. Genet. 37: 495-500.

Lall, S., Grun, D., Krek, A., Chen, K., Wang, Y.L., Dewey, C.N., Sood, P., Colombo, T., Bray, N., Macmenamin, P., et al. 2006. A genome-wide map of conserved microRNA targets in C. elegans. Curr. Biol. 16: $460-471$.

Landgraf, P., Rusu, M., Sheridan, R., Sewer, A., Iovino, N., Aravin, A., Pfeffer, S., Rice, A., Kamphorst, A.O., Landthaler, M., et al. 2007. A mammalian microRNA expression atlas based on small RNA library sequencing. Cell 129: 1401-1414.

Lewis, B.P., Shih, I.H., Jones-Rhoades, M.W., Bartel, D.P., and Burge, C.B. 2003. Prediction of mammalian microRNA targets. Cell 115: 787-798.

Lewis, B.P., Burge, C.B., and Bartel, D.P. 2005. Conserved seed pairing, often flanked by adenosines, indicates that thousands of human genes are microRNA targets. Cell 120: 15-20.

Lim, L.P., Lau, N.C., Garrett-Engele, P., Grimson, A., Schelter, J.M. Castle, J., Bartel, D.P., Linsley, P.S., and Johnson, J.M. 2005. Microarray analysis shows that some microRNAs downregulate large numbers of target mRNAs. Nature 433: 769-773.

Majoros, W.H. and Ohler, U. 2007. Spatial preferences of microRNA targets in 3' untranslated regions. BMC Genomics 8: 152. doi: 10.1186/1471-2164-8-152.

Mallory, A.C., Reinhart, B.J., Jones-Rhoades, M.W., Tang, G., Zamore, P.D., Barton, M.K., and Bartel, D.P. 2004. MicroRNA control of PHABULOSA in leaf development: Importance of pairing to the

\section{Genome Research}

www.genome.org 
microRNA 5' region. EMBO J. 23: 3356-3364.

Miller, W., Rosenbloom, K., Hardison, R.C., Hou, M., Taylor, J., Raney,

B., Burhans, R., King, D.C., Baertsch, R., Blankenberg, D., et al. 2007. 28-way vertebrate alignment and conservation track in the UCSC Genome Browser. Genome Res. 17: 1797-1808.

Nielsen, C.B., Shomron, N., Sandberg, R., Hornstein, E., Kitzman, J., and Burge, C.B. 2007. Determinants of targeting by endogenous and exogenous microRNAs and siRNAs. RNA 13: 1894-1910.

Pruitt, K.D., Tatusova, T., and Maglott, D.R. 2005. NCBI Reference Sequence (RefSeq): A curated non-redundant sequence database of genomes, transcripts and proteins. Nucleic Acids Res. 33: D501-D504. doi: 10.1093/nar/gkl842.

Rodriguez, A., Vigorito, E., Clare, S., Warren, M.V., Couttet, P., Soond, D.R., van Dongen, S., Grocock, R.J., Das, P.P., Miska, E.A., et al. 2007. Requirement of bic/microRNA-155 for normal immune function. Science 316: 608-611.

Ruby, J.G., Jan, C., Player, C., Axtell, M.J., Lee, W., Nusbaum, C., Ge, H., and Bartel, D.P. 2006. Large-scale sequencing reveals 21U-RNAs and additional microRNAs and endogenous siRNAs in C. elegans. Cell 127: 1193-1207.

Ruby, J.G., Stark, A., Johnston, W.K., Kellis, M., Bartel, D.P., and Lai, E.C. 2007. Evolution, biogenesis, expression, and target predictions of a substantially expanded set of Drosophila microRNAs. Genome Res. 17: 1850-1864.

Selbach, M., Schwanhäusser, B., Thierfelder, N., Fang, Z., Khanin, R., and Rajewsky, N. 2008. Widespread changes in protein synthesis induced by microRNAs. Nature 455: 58-63.

Stark, A., Brennecke, J., Bushati, N., Russell, R.B., and Cohen, S.M. 2005. Animal microRNAs confer robustness to gene expression and have a significant impact on 3'UTR evolution. Cell 123: 1133-1146.

Stark, A., Kheradpour, P., Parts, L., Brennecke, J., Hodges, E., Hannon, G.J., and Kellis, M. 2007a. Systematic discovery and characterization of fly microRNAs using 12 Drosophila genomes. Genome Res. 17: 1865-1879.

Stark, A., Lin, M.F., Kheradpour, P., Pedersen, J.S., Parts, L., Carlson, J.W., Crosby, M.A., Rasmussen, M.D., Roy, S., Deoras, A.N., et al. $2007 b$. Discovery of functional elements in 12 Drosophila genomes using evolutionary signatures. Nature 450: 219-232.

Vella, M.C., Choi, E.Y., Lin, S.Y., Reinert, K., and Slack, F.J. 2004. The C. elegans microRNA let-7 binds to imperfect let-7 complementary sites from the lin-41 3'UTR. Genes \& Dev. 18: 132-137.

Wolfe, K.H., Sharp, P.M., and Li, W.H. 1989. Mutation rates differ among regions of the mammalian genome. Nature 337: 283-285.

$\mathrm{Wu}$, L. and Belasco, J.G. 2005. Micro-RNA regulation of the mammalian lin-28 gene during neuronal differentiation of embryonal carcinoma cells. Mol. Cell. Biol. 25: 9198-9208.

Xie, X., Lu, J., Kulbokas, E.J., Golub, T.R., Mootha, V., Lindblad-Toh, K., Lander, E.S., and Kellis, M. 2005. Systematic discovery of regulatory motifs in human promoters and 3'UTRs by comparison of several mammals. Nature 434: 338-345.

Yang, Z. and Bielawski, J.P. 2000. Statistical methods for detecting molecular adaptation. Trends Ecol. Evol. 15: 496-503.

Yekta, S., Shih, I.H., and Bartel, D.P. 2004. MicroRNA-directed cleavage of HOXB8 mRNA. Science 304: 594-596.

Received June 27, 2008; accepted in revised form October 15, 2008. 


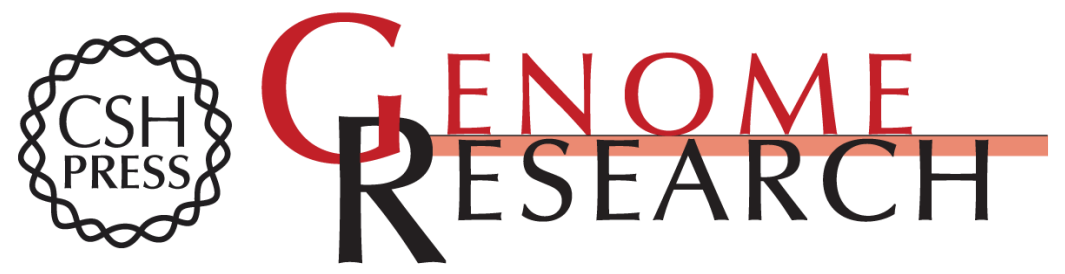

\section{Most mammalian mRNAs are conserved targets of microRNAs}

Robin C. Friedman, Kyle Kai-How Farh, Christopher B. Burge, et al.

Genome Res. 2009 19: 92-105 originally published online October 27, 2008

Access the most recent version at doi:10.1101/gr.082701.108

Supplemental http://genome.cshlp.org/content/suppl/2008/12/03/gr.082701.108.DC1
Material

References This article cites 38 articles, 14 of which can be accessed free at: http://genome.cshlp.org/content/19/1/92.full.html\#ref-list-1

Open Access Freely available online through the Genome Research Open Access option.

License Freely available online through the Genome Research Open Access option.

Email Alerting Receive free email alerts when new articles cite this article - sign up in the box at the Service top right corner of the article or click here.

\section{Affordable, Accurate} Sequencing. 